\title{
Phased array antenna for millimeter-wave application
}

\author{
O. Nayat-Ali ${ }^{1}$, O. El Mrabet ${ }^{1}$, M. Aznabet ${ }^{1}$, F.Z. Khoutar ${ }^{1}$ \\ \{Oumaima.nayatali@gmail.com ${ }^{1}$, O.mrabet@gmail.com ${ }^{1}$, mariamaznabet@gmail.com ${ }^{1}$ \} \\ Information and Telecommunication Systems Lab ${ }^{1}$, Faculty of Sciences, University of Abdelmalek \\ Essaadi, Tetuan, Morocco, fs.tetouan@gmail.com
}

\begin{abstract}
Beam-steering antenna is essential and widely used in communication system. The design of $28 \mathrm{GHz}$ beam steering phased array antenna for $5 \mathrm{G}$ millimeterwave application presented in this communication. The objective of this research is to investigate and implement a phase shifter in a linear antenna array for angle scan. The proposed array antenna has four microstrip patch antenna elements, each feed ports are connected to phase shifter. The main mechanism of radiation is due to the excitation of element in sequence with different phase delays. The overall radiated beam can be steered to different directions depending on the initial excitation of elements. In the first, 3 -D good radiation by $0^{\circ}$ phase. In the second, good scanning performance of a range of $\pm 10^{\circ}$ to $\pm 40^{\circ}$, acceptable side lobe level and gain of $12.1 \mathrm{~dB}$ are obtained.
\end{abstract}

Keywords: Linear Array; Phase Shifters; Phased Array; Radiation Pattern; Microstrip Antenna; Beam Direction.

\section{Introduction}

In this paper, millimeter-wave communication has become a key enabler for $5 \mathrm{G}$ application.

Phased array antennas can adopt a number of different configurations, including linear, planar, and circular. Phased array antennas are used mainly to steer the main beam (main lobe, broadside) of the antenna electronically. It operates by delaying signals coming out of antenna elements by using phase shifter on each antenna element. This provides constructive and destructive interference, thus giving the result that the beam is steered away from the nominal (main) direction of an individual antenna element [1],[6].

This work focuses on linear phased array antennas whose unit cell is formed by a radiating elements, each fed by a transmit and receive module that can provide amplitude and phase control. Linear phased arrays use the progressive phase excitation between the elements to scan the antenna beam electronically over one dimension. The main advantages offered by phased arrays over conventional systems are increased scanning speed, high reliability, and multifunction capability. These advantages make the use of this technology the most logical choice for the next generation weather radars. The use of linear active phased arrays as a component of future low-cost weather radars is the major motivator of this work; consequently, this paper is dedicated to explain the basic concepts related to the theory of linear phase arrays and how their characteristics can be used in the antennas [2]. 
The organization of the paper is as follows: Section II describes the phased array antenna principle. Section III discusses issues related to the proposed patch antenna design and simulation results. Section IV presents phased array antenna proposed structure and finally the conclusion.

\section{Phased array antenna}

In single element antenna, the radiation pattern is usually very broad and the directivity is relatively low. This problem can be affected by enlarging the size of the element thus increasing the directivity. Another way to enlarge the antenna without changing the size of the individual elements is to assemble the radiating elements in a geometrical configuration known as an "array" [5]. The individual elements forming the array are usually identical and they can be of any form (wire antenna, microstrip patches etc...). Many antenna system applications require that the direction of the beam's main lobe be changed with time, or scanned. This is usually done by mechanically rotating a single antenna or an array with fixed phase to the element. However, mechanical scanning requires a positioning system that can be costly and scan too slowly. For this reason, electronic scanning antennas which are known as phased array antennas are used. It can sweep the direction of the beam by varying electronically the phase of the radiating element, there by producing a moving pattern with no moving parts. Phased array antennas are known for their capability to steer the beam pattern electronically with high effectiveness, managing to get minimum side-lobe level and narrow beamwidth [7]. To achieve performance specifications such as narrow beamwidth or considerable scanning range with high angle resolution, a large number of antenna elements were needed to construct the array. Normally phase shifters are the devices in a phased array antenna that allow the radiated beam to be steered in the direction [4].

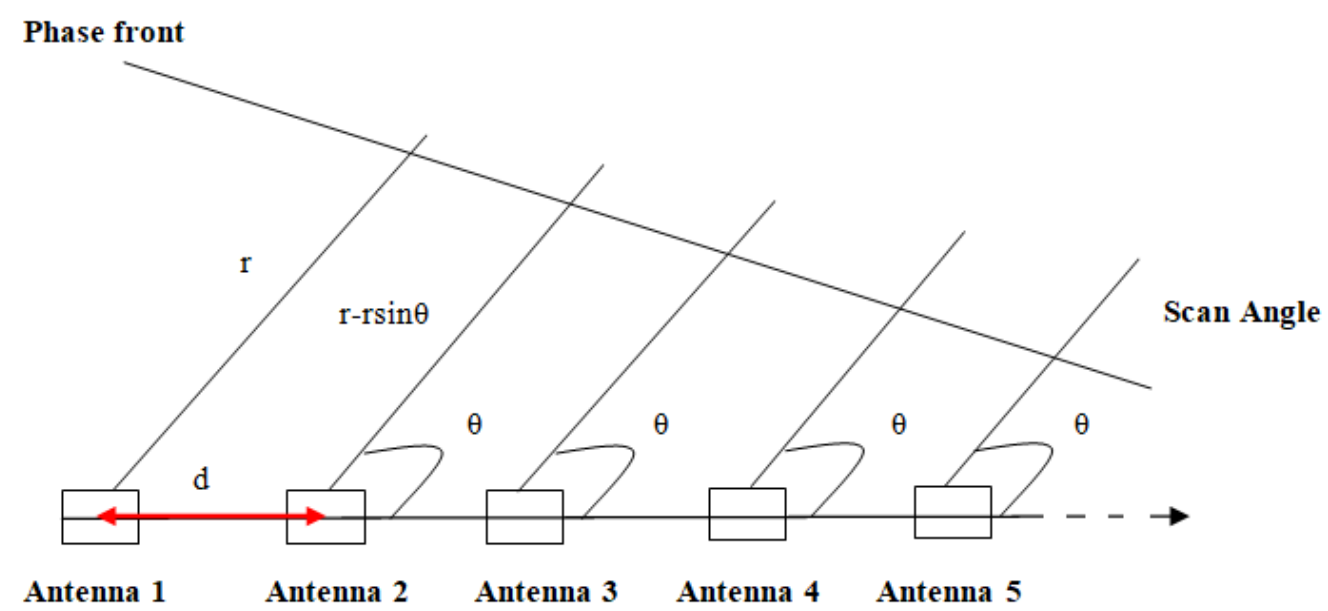

Fig. 1. Uniform linear array of $\mathrm{N}$ elements. 
In Figure 1. shows a uniform linear array of $\mathrm{N}$ equally spaced elements having a grating step (separating two adjacent antennas). These sources are fed with the same amplitude and with a phase gradient $\Delta \varphi$ with respect to each other. $r$ represents the maximum distance between the reference antenna and the observation plane, and $\theta$ is the beam steering angle. (1):

In these conditions, the total electric field radiated by the array antenna is then written as

Where:

$$
\mathrm{E}_{\mathrm{tot}}=\mathrm{E}_{0} * \mathrm{~A}(\psi)
$$

- $\mathrm{E}_{0}$ represents the electric field radiated by an elementary antenna. It is named "element factor".

- A $(\psi)$ is the "Array factor» independent of the elementary source that makes up the array.

This array factor is given by the relation (2) below:

Where,

$$
\mathrm{A}(\psi)=\frac{1}{N} \exp (\mathrm{j}(\mathrm{N}-1) \psi / 2) \frac{\sin \left(\frac{N \psi}{2}\right)}{\sin \left(\frac{\psi}{2}\right)} \text {. }
$$

$\Psi=\frac{2 \pi d}{\lambda} \sin (\theta)-\Delta \phi$, and $\lambda$ is the wavelength

The total radiation pattern of the grating antenna is defined by the product of the modulus of the electric field radiated by an elementary antenna by that of the grating factor, the latter being given by the following expression see equation (3):

$$
|A(\psi)|=\frac{1}{N}\left|\frac{\sin \left(\frac{N \psi}{2}\right)}{\sin \left(\frac{\psi}{2}\right)}\right| .
$$

In these conditions, it should be noted that as soon as the number $\mathrm{N}$ of elementary antennas becomes large, the total radiation pattern of the array depends essentially on the array factor and very little of the radiation pattern of each antenna. Note that if all the sources are excited in phases ( $\psi$ is zero), the radiated field is maximum in the plane normal to the array.

Conversely, if the sources are excited with a non-zero phase gradient, the maximum radiation appears for $\psi=2 \mathrm{~m} \pi$. The solutions are periodic and show on the same array lobes radiation pattern for values of $m \neq 0$.

Thus, a variation of the phase gradient of the array $\Delta \varphi$ to be introduced between the adjacent elementary sources causes the orientation of the radiation pattern in the desired $\theta 0$ direction according to:

$$
\Delta \varphi=\frac{2 \pi d}{\lambda} \sin \left(\theta_{0}\right)
$$

The variation of the phase gradient of the array determines that of the beam steering angle $\theta 0$ of the array antenna: this is the principle of a "phased array antenna"[3].

\section{Proposed antenna}

The geometry of the proposed single-patch antenna structures is shown in Figure 2. consists of a the conventional square patch fed with the transmission line structure printed on a Rogers RT/Duroid5880 substrate, which has permittivity of 2.2, loss tangent of 0.001 and having a thickness of $\mathrm{h}=0.25 \mathrm{~mm}$. The area of the proposed antenna is $10 \times 15 \mathrm{~mm}^{2}$ and the dimensions of the antenna are calculated and optimized so that it resonates at $28 \mathrm{GHz}$ 
frequency using patch antenna equations, the final dimensions of the proposed antenna are: $\mathrm{L}_{\mathrm{p}}=\mathrm{W}_{\mathrm{p}}=3.36 \mathrm{~mm}, \mathrm{~L}_{\mathrm{q}}=2 \mathrm{~mm}, \mathrm{~W}_{\mathrm{q}}=0.145 \mathrm{~mm}, \mathrm{~L}_{\mathrm{f}}=3 \mathrm{~mm}$ and $\mathrm{W}_{\mathrm{f}}=0.776 \mathrm{~mm}$.

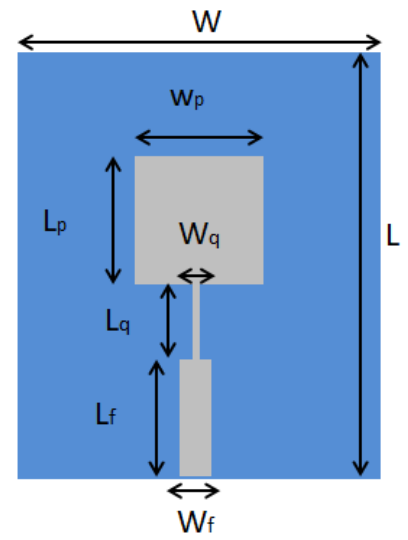

Fig. 2. Geometry of the proposed patch antenna.

\subsection{Simulation results and discussions}

The proposed antenna is simulated using CST Microwave StudioTM and the calculated Sparameter is shown in Figure 3. The proposed structure gives return loss around $-19.5 \mathrm{~dB}$ at the resonant frequency $28 \mathrm{GHz}$ and has a $27.21-28.30 \mathrm{GHz}$ bandwidth for $|\mathrm{S} 11|<-10 \mathrm{~dB}$, which is approximately $3.69 \%$ bandwidth $(1.09 \mathrm{GHz})$ with a center frequency of $28 \mathrm{GHz}$.

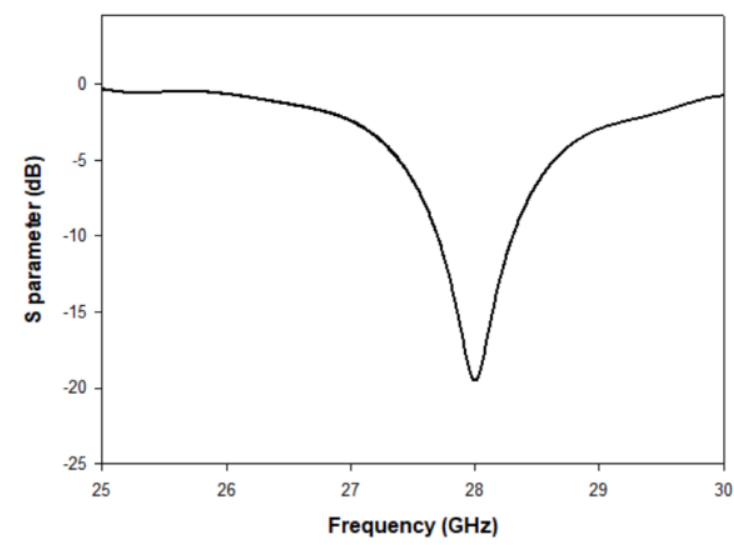

Fig. 3. Simulated result of $S$ parameter of the initial patch.

The Figure 4. explains the antenna radiation pattern. The antenna exhibits a maximum gain of $7.32 \mathrm{~dB}$. The Side lobe level for the E-plane and H-plane are $-20.5 \mathrm{~dB}$ and $-12.9 \mathrm{~dB}$ respectively. Half power beamwidth of $87.3^{\circ}$ is obtained H-plane, whereas that of E-plane is $76^{\circ}$. 


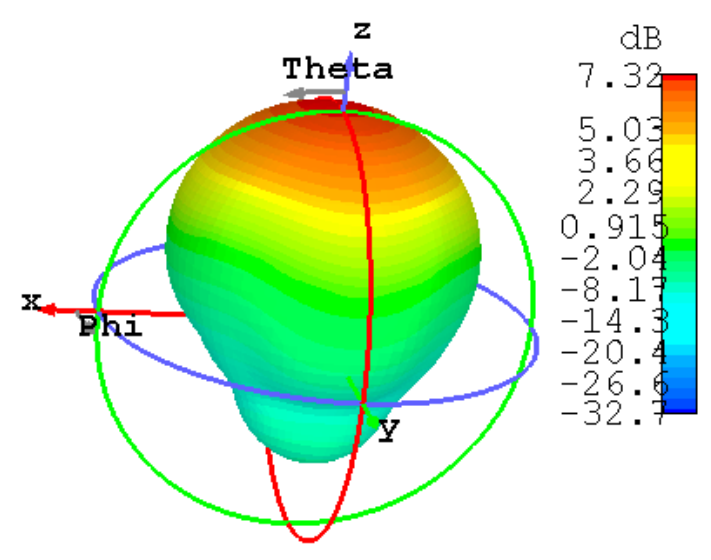

Fig. 4. Simulated 3D Far-field Radiation Pattern at $28 \mathrm{GHz}$.

\subsection{Array of two element microstrip patch}

Figure 5. shows an array of two element patch antenna separated by a distance $d$ that does varies between $0.5 \lambda$ and $0.8 \lambda$. The goal of this study is to optimize the separation distance between the two elements of the array in order to find a compromise between the side lobe level and the isolation between the two excitation ports. The results found are grouped in the Table 1:

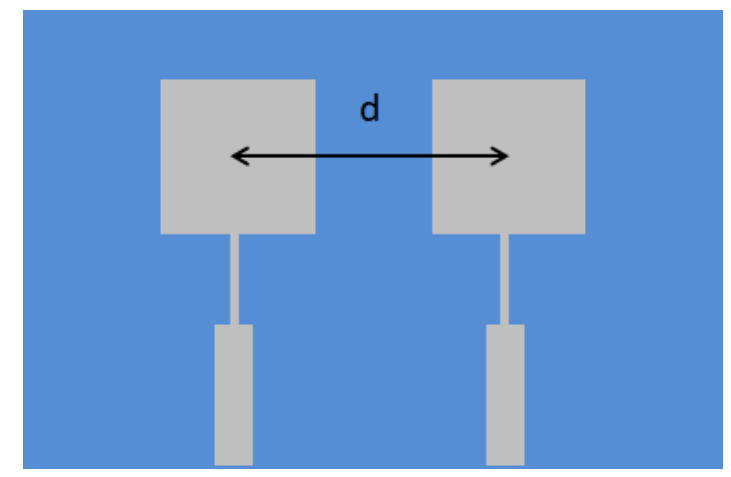

Fig. 5. Array of two element microstrip patch.

Table 1. The effect of separated disance $\mathrm{d}$ between the element on the antenna performance.

\begin{tabular}{cccc}
\hline $\begin{array}{c}\text { Separated distance } \\
\mathrm{d}\end{array}$ & $\mathrm{S} 21(\mathrm{~dB})$ & Gain $(\mathrm{dB})$ & SLL $(\mathrm{dB})$ \\
\hline $0.5 \lambda$ & & & \\
$0.6 \lambda$ & -26.7 & 10.5 & -14.7 \\
$0.7 \lambda$ & -28.84 & 7.58 & -18.4 \\
$0.8 \lambda$ & -30.41 & 7.95 & -17.7 \\
\hline
\end{tabular}


We can see that the greater the separation distance between these two elements, the better isolation and inferior gain. However, there are a secondary lobes with high gain that are considered parasitic radiation. To reduce the side lobes level, we have to bring closer the antennas as possible. However, the closer they are the closer the field to antenna coupling is important. It was therefore necessary to find a compromise between the separation distance for the reduction of the side lobe level and the coupling between antennas. Based on this analysis, we chose as optimal separations distance $0.5 \lambda$ because for this the array has good performances at the side lobes level, coupling between antennas and gain of antenna.

\section{Linear array antenna}

Phased array antennas are known for their capability to steer the beam pattern electronically with high effectiveness, managing to get minimum side-lobe levels and narrow beamwidths. Normally phase shifters are the devices in a phased array antenna that allow the radiated beam to be steered in the direction [4]. The objective of this part is to investigate a phase shifter in a linear antenna array for angle scan. Figure 6. depicts a linear array of 4 elements; all elements are excited with equal amplitude and with a progressive phase shift $\Delta \varphi$ determined by the location of the element in the array.

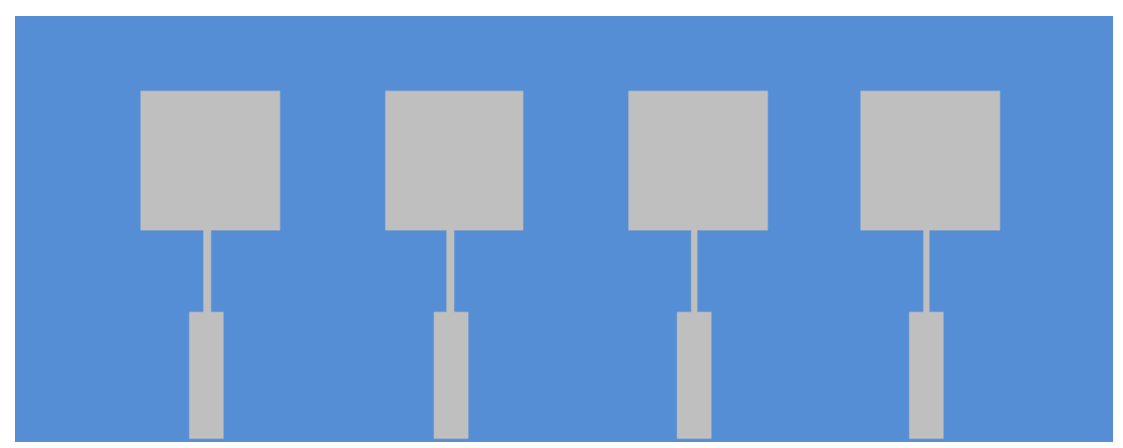

Fig. 6. Array of four element microstrip patch.

The return loss of the antenna is shown in Figure 7. From the result the resonance frequency of the antenna is at $28.03 \mathrm{GHz}$ and $\mathrm{S} 11=\mathrm{S} 22=-16.17 \mathrm{~dB}$ and $\mathrm{S} 33=\mathrm{S} 44=-14.78 \mathrm{~dB}$. The bandwidth of the antenna is $2.03 \%$. 


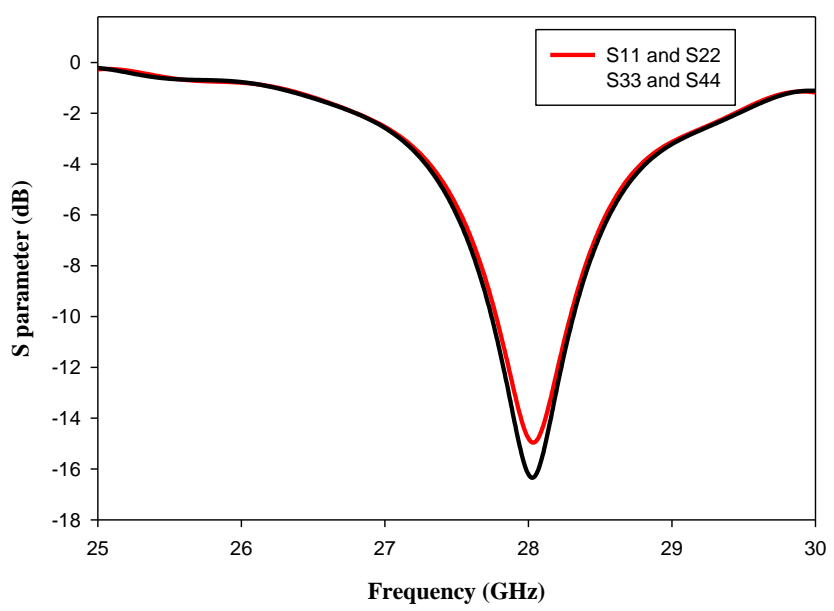

Fig. 7. Array of four element microstrip patch.

In the first, all the ports are excited by 0 phase and equal amplitude, the results of the radiation patterns in (XY) E-plane and properties such as beam position, maximum gain and SLL are shown in Figure 8. The maximum gain equal to $12 \mathrm{~dB}$ in broadside $\left(\theta=0^{\circ}\right)$. Here, the SLL is equal to $-13.5 \mathrm{~dB}$ at E-plane. In the second, to study the beam scanning capability of the array antenna all the ports are excited by a scanning over $\pm 40^{\circ}$ and equal amplitude. In Figure 9. the radiation pattern for (XY) E-plane for different values of phase from $-40^{\circ}$ to $40^{\circ}$ at resonance frequency $28 \mathrm{GHz}$ are shown. The gain for all the scanning is $12 \mathrm{~dB}$, but the side lobe level is $-10.8 \mathrm{~dB}$ when the beam is tilted by $\pm 30^{\circ}$ and $\pm 40^{\circ}$, and its gain remains constant $12 \mathrm{~dB}$. When we have the beam tilting of $\pm 20^{\circ}$ gets a SLL of $-11.5 \mathrm{~dB}$ at $28 \mathrm{GHz}$ and beamwidth of $26.3^{\circ}$.

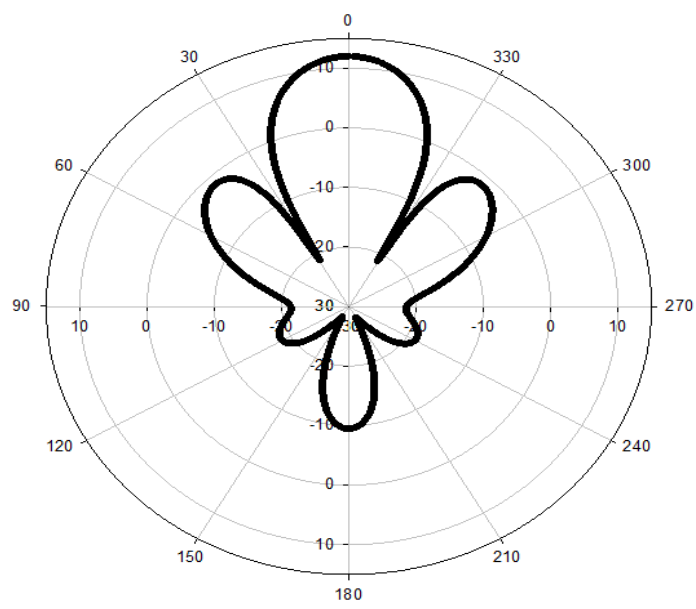

Fig. 8. Simulated radiation pattern For $(X Y)$ E-plane $\Phi=0$ Phase $0^{\circ}$. 


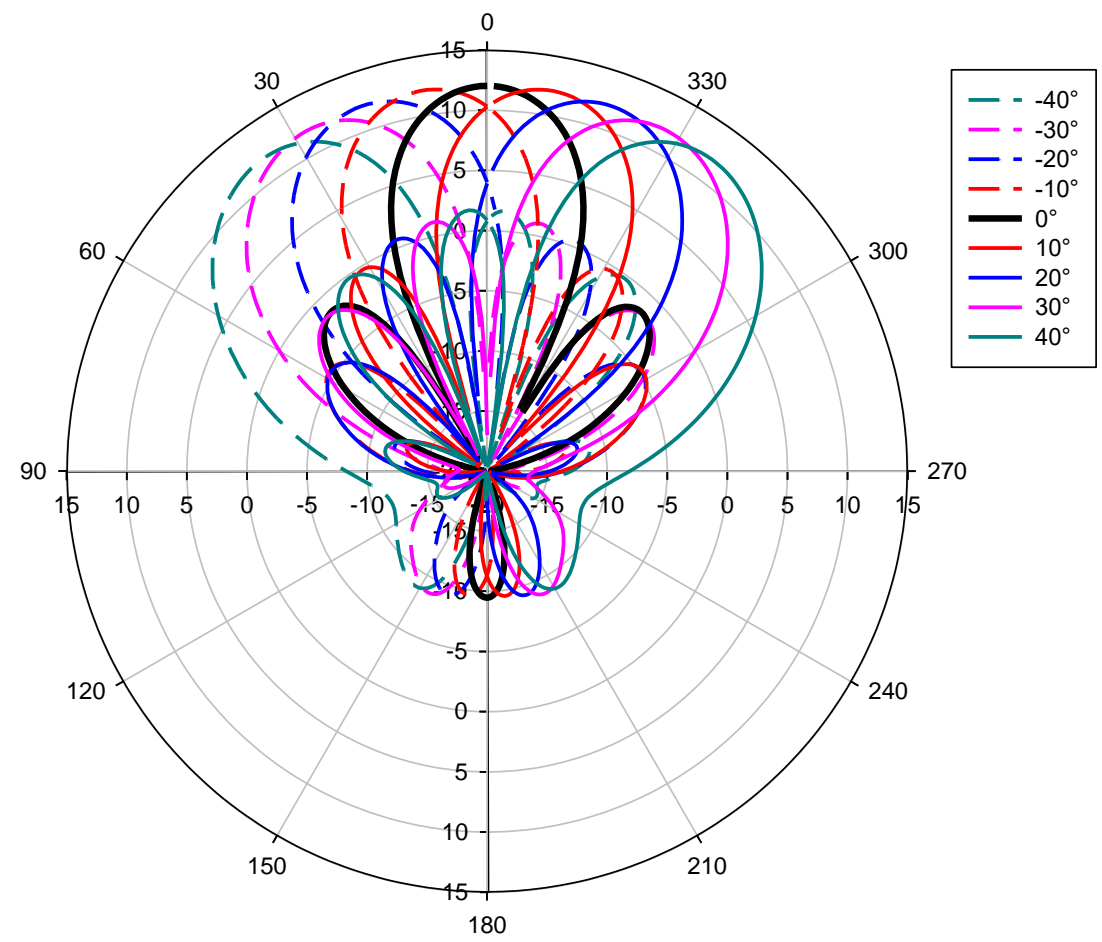

Fig. 9. Simulated radiation pattern For $(X Y)$ E-plane $\Phi=0$ Phase \pm 10 to \pm 40 .

\section{Comparison with literature}

As indicated at the onset of the paper, a number of techniques have been proposed in the literature for the beam steering. Table 2 presents a comparison of the scanning angular range of the proposed antenna and the beam steering antennas in [6], [8] and [9]. We can see that the proposed antenna realizes a larger beam tilted angle.

Table 2. Comparison of techniques reported in literature for beam steering.

\begin{tabular}{|c|c|c|c|}
\hline Antenna Model & Fr $(\mathrm{GHz})$ & Beam Tilt $\left({ }^{\circ}\right)$ & Technology \\
\hline Antenna in [6] & 28 & \pm 25 & $\begin{array}{c}\text { Series-Fed Pach } \\
\text { Antennas }\end{array}$ \\
\hline Antenna in [8] & 34.25 & \pm 30 & $\begin{array}{l}\text { Antenna Using a } \\
\text { Metasurface }\end{array}$ \\
\hline Antenna in [9] & 9 & \pm 30 & $\begin{array}{c}\text { Design Of Phase- } \\
\text { Modulated } \\
\text { Metasurfaces }\end{array}$ \\
\hline $\begin{array}{l}\text { Antenna in this } \\
\text { paper }\end{array}$ & 28 & \pm 40 & $\begin{array}{c}\text { Uniform linear } \\
\text { array }\end{array}$ \\
\hline
\end{tabular}




\section{Conclusion}

In this paper, a phased array structure has been proposed and analyzed. The structure is formed by uniformly arranged as an 4-element identical, which is able to achieve beam steering with using phase shifters. The phase shift are achieved by changing the phase of the element. The final proposed antenna achieves a $750 \mathrm{MHz}$ bandwidth for $\mathrm{S} 11<-10 \mathrm{~dB}$ to cover 27.74-28.31 GHz frequency band which is intended for future millimeter wave communications. Moreover, the antenna array achieved a maximum gain of $12 \mathrm{~dB}$ in the broadside direction. The antenna array could be utilized as a phased array and hence could be used to steer the radiation beam. The antenna exhibited a very good radiation pattern and stable gain. The structure can have 9 kinds of reflection phase distribution, so the antenna can change its beams among $0^{\circ}, \pm 10^{\circ}, \pm 20^{\circ}, \pm 30^{\circ}$ and $\pm 40^{\circ}$ in xoy-plane at $28 \mathrm{GHz}$. Compare to other beam steering antenna, the proposed antenna can realize a larger beam tilted angle $\left(40^{\circ}\right)$. The next step is to fabricate the antenna and verify the simulation results which will be reported at the conference.

\section{References}

[1] D. Ehyaie: Novel Approaches to the Design of Phased Array Antennas. pp. 1-11 2011.

[2] T. Eray, H. Jurgen,W. Christoph, Z. Thomas: A novel millimeter-wave dual-fed phased array for beam steering. IEEE transaction on microwave theory and technique. Vol. 61, pp. 40-43. 2013.

[3] M. Dorra: Etude comportementale et conception d'un réseau d'oscillateurs couplés intégré en technologie silicium appliqué à la commande d'un réseau d'antennes linéaire.2013.

[4] L. Jinxin, Z. Qingsheng, L. Ruizhi, A. Tayeb: Beam-tilting antenna with negative refractive indexe metamateriam loading. IEEE antennas and wirless propagation letters. Vol. 61, pp. 40-43. 2017.

[5] Y. Yazid, G. Xun: A low-cost patch antenna phased array with analog beam steering using mutual coupling and reactive loading. IEEE antenna and wirless propagation letters. Vol. 7. 2012.

[6] M. Khalily, R. Tafazolli, T. A. Rahman, and M. R. Kamarudin: Design of phased arrays of seriesfed patch antennas with reduced number of the controllers for $28 \mathrm{GHz} \mathrm{mm}$-wave applications. IEEE Antennas and Wireless Propagation Letters, vol. 15, pp. 1305-1308, 2016.

[7] T. Eray, H. Jurgen, W. Christoph, Z. Thomas: Reconfigurable Beam Steering Using a Microstrip Patch Antenna With a U-Slot for Wearable Fabric Applications. IEEE antennas and wirless propagation letters. Vol. 15,2013.

[8] L. Teng, Z. N. Chen: Control of Beam Direction for Substrate-Integrated Waveguide Slot Array Antenna Using Metasurface. IEEE Transaction On Antennas And Propagation. 2018.

[9] R. Badreddine, L. Andre, P. Gerard, N. Shah: Modeling and design of metasurfaces for beam scanning. Appl. Phys. Lett. 50, 123,2017. 